\title{
Conserved, divergent and heterochronic gene expression during Brachypodium and Arabidopsis embryo development
}

\author{
Zhaodong Hao $^{1,2} \cdot$ Zhongjuan Zhang $^{1} \cdot$ Daoquan Xiang $^{3} \cdot$ Prakash Venglat $^{4} \cdot$ Jinhui Chen $^{2} \cdot$ Peng Gao $^{5} \cdot$ Raju Datla $^{5}$. \\ Dolf Weijers ${ }^{1}$ (i)
}

Received: 5 March 2021 / Accepted: 23 April 2021 / Published online: 5 May 2021

(c) The Author(s) 2021

\begin{abstract}
Key message Developmental and transcriptomic analysis of Brachypodium embryogenesis and comparison with Arabidopsis identifies conserved and divergent phases of embryogenesis and reveals widespread heterochrony of developmental gene expression.

Abstract Embryogenesis, transforming the zygote into the mature embryo, represents a fundamental process for all flowering plants. Current knowledge of cell specification and differentiation during plant embryogenesis is largely based on studies of the dicot model plant Arabidopsis thaliana. However, the major crops are monocots and the transcriptional programs associated with the differentiation processes during embryogenesis in this clade were largely unknown. Here, we combined analysis of cell division patterns with development of a temporal transcriptomic resource during embryogenesis of the monocot model plant Brachypodium distachyon. We found that early divisions of the Brachypodium embryo were highly regular, while later stages were marked by less stereotypic patterns. Comparative transcriptomic analysis between Brachypodium and Arabidopsis revealed that early and late embryogenesis shared a common transcriptional program, whereas mid-embryogenesis was divergent between species. Analysis of orthology groups revealed widespread heterochronic expression of potential developmental regulators between the species. Interestingly, Brachypodium genes tend to be expressed at earlier stages than Arabidopsis counterparts, which suggests that embryo patterning may occur early during Brachypodium embryogenesis. Detailed investigation of auxin-related genes shows that the capacity to synthesize, transport and respond to auxin is established early in the embryo. However, while early PIN1 polarity could be confirmed, it is unclear if an active response is mounted. This study presents a resource for studying Brachypodium and grass embryogenesis and shows that divergent angiosperms share a conserved genetic program that is marked by heterochronic gene expression.
\end{abstract}

Keywords Brachypodium distachyon $\cdot$ Embryogenesis $\cdot$ RNA-seq

Communicated by Thomas Dresselhaus.

Dolf Weijers

dolf.weijers@wur.nl

1 Laboratory of Biochemistry, Wageningen University, Stippeneng 4, Wageningen, The Netherlands

2 Key Laboratory of Forest Genetics \& Biotechnology of Ministry of Education, Co-Innovation Center for Sustainable Forestry in Southern China, Nanjing Forestry University, Nanjing, Jiangsu, China

3 Aquatic and Crop Resource Development, National Research Council Canada, Saskatoon, SK, Canada

4 Department of Plant Sciences, College of Agriculture, University of Saskatchewan, Saskatoon, SK, Canada

5 Global Institute for Food Security, University of Saskatchewan, Saskatoon, SK, Canada

\section{Introduction}

Angiosperms represent a diverse group of plants that share a number of characteristics: a dominant diploid sporophytic state, true embryos with precursors for the major tissues, including meristems, an elaborate vascular transport system, seeds and flowers. Both major groups of angiosperms: dicots and monocots, encompass crops as well as genetic model organisms. In both groups, the embryo represents a relatively simple form in which-from a fertilized egg cell-a miniature plant emerges that has primordial organs and tissues, including meristems that sustain post-embryonic growth. Few models have been used to extensively study progression and genetics of embryo development, and these include the dicots tobacco, Arabidopsis thaliana and soybean, as well 
as the monocots rice, maize and wheat (Armenta-Medina et al. 2021; Palovaara et al. 2016). From these analyses, as well as from earlier comparative embryology (Johri 1984), it is evident that the morphology and developmental progression is very different between dicots and monocots. In fact, it is difficult to even identify homologous stages based on morphology. Thus, whereas there is a prominent body of literature on genetic regulation of Arabidopsis embryogenesis (reviewed in Palovaara et al. 2016; ten Hove et al. 2015), it is far from trivial to transpose this toward monocot plants (Zhao et al. 2017). Following the identification of developmental regulators in Arabidopsis, analysis of expression patterns of maize or rice homologs has shown that there is both conservation and divergence of expression patterns. For example, within the WOX family, some members show different patterns between Arabidopsis and maize (Haecker et al. 2004; Nardmann et al. 2007), while the pattern of WOX5 appears conserved between Arabidopsis, maize and rice (Kamiya et al. 2003; Nardmann et al. 2007; Sarkar et al. 2007). Likewise, the Arabidopsis STM and maize $K N$ genes have similar expression (Kerstetter et al. 1997; Long and Barton 1998; Smith et al. 1995). Thus, a major open question is how (dis)similar embryo developmental patterns and their regulation are between monocots and dicots.

Several studies have focused on monocot embryogenesis from either a morphological (Black et al. 2006; Guillon et al. 2012; Itoh et al. 2005; Smart and O'Brien 1983) or transcriptional (Chen et al. 2017; Itoh et al. 2016; Yi et al. 2019) perspective. From these, however, it is not yet clear how the developmental transitions and emergence of pattern elements are connected to genome-wide gene expression patterns. At the same time, it is not yet clear how the transcriptional landscape of monocot embryogenesis relates to that found in dicots.

Here, we focus on the development of the Brachypodium distachyon embryo. Brachypodium is a monocot grass model plant (Scholthof et al. 2018) that is closely related to wheat, yet is diploid, has a small size and short life cycle that allows cultivation in laboratory conditions and has not been domesticated. Thus, it represents a "wild" grass model. The Brachypodium genome has been sequenced (The International Brachypodium Initiative 2010), and the species is being used as model for bioenergy (Cass et al. 2016), root development (Agapit et al. 2020) and flowering (Qin et al. 2017), among others. Given that closely related crop relatives, such as wheat, are seed crops, there is an interest in understanding the control of embryo and grain development. An initial description of grain development (Guillon et al. 2012) showed that general developmental patterns of embryo development are comparable between Brachypodium and other grasses.

Here, we combined detailed analysis of cell division patterns with stage-specific transcriptome analysis to provide insights into Brachypodium embryogenesis. By comparative transcriptomics, we find that the early and late embryo phases share genetic programs between Brachypodium and Arabidopsis, whereas mid-embryogenesis is divergent. Analysis of orthology groups reveals widespread heterochrony of embryo development, where Brachypodium appears to express many genes at earlier stages than the Arabidopsis counterpart. Detailed investigation of auxin transport and response shows conserved expression between species, but it is unclear if the hormone controls embryogenesis in Brachypodium. Thus, embryogenesis in Brachypodium is marked by a conserved angiosperm transcriptional program, as well as lineage-specific programs and heterochronic expression of many potential regulators.

\section{Results}

\section{Brachypodium distachyon embryo development}

Embryo development of several grass species have been described. Generally, embryo stages are comparable between Brachypodium and wheat (Guillon et al. 2012; Xiang et al. 2019). Here, we extended earlier descriptions of embryogenesis with an emphasis on early, morphogenetic stages. Through whole-mount microscopy and scanning electron microscopy, we confirmed the previously described stages and here systematically name these as two-cell embryo or quadrant (TCQ; Fig. 1A), pro-embryo (PEM; Fig. 1B), transition (TRA; Fig. 1C, D), leaf early (LEE; Fig. 1E-G), leaf middle (LEM; Fig. 1H, I), leaf late (LEL; Fig. 1J, K) and mature (MAT; Fig. 1L, M). For the earliest stages, we additionally performed ClearSee-based staining (Ursache et al. 2018), followed by high-resolution confocal microscopy (Fig. 1N-U) and cell segmentation (Fig. 2) (Yoshida et al. 2014). In the following, we describe the morphogenetic hallmarks of embryo progression and its cellular basis.

Analysis of the earliest stages of embryogenesis revealed that zygote division is asymmetric (Figs. $1 \mathrm{~N}$ and 2A), generating a small apical and large basal cell. Following this initial, asymmetric division, each of the daughter cells again divides asymmetrically, generating two neighboring small cells (Fig. 2B, C). While the following divisions were less stereotypical, the pattern of divisions generated a cluster of small cells, likely from the initial two small daughter cells, surrounded by a group of larger cells (Fig. 2D, E). At early stages, the embryo thus already displays the laterally bent structure that characterizes later stages. From the lateral region of larger cells, the scutellum originates, while a dome that marks the shoot apical meristem (SAM) area arises underneath the scutellum (Fig. 1R, S, V). An axis of smaller cells extends basally from the SAM, but distinct tissues, such as the future root meristem, are not readily 


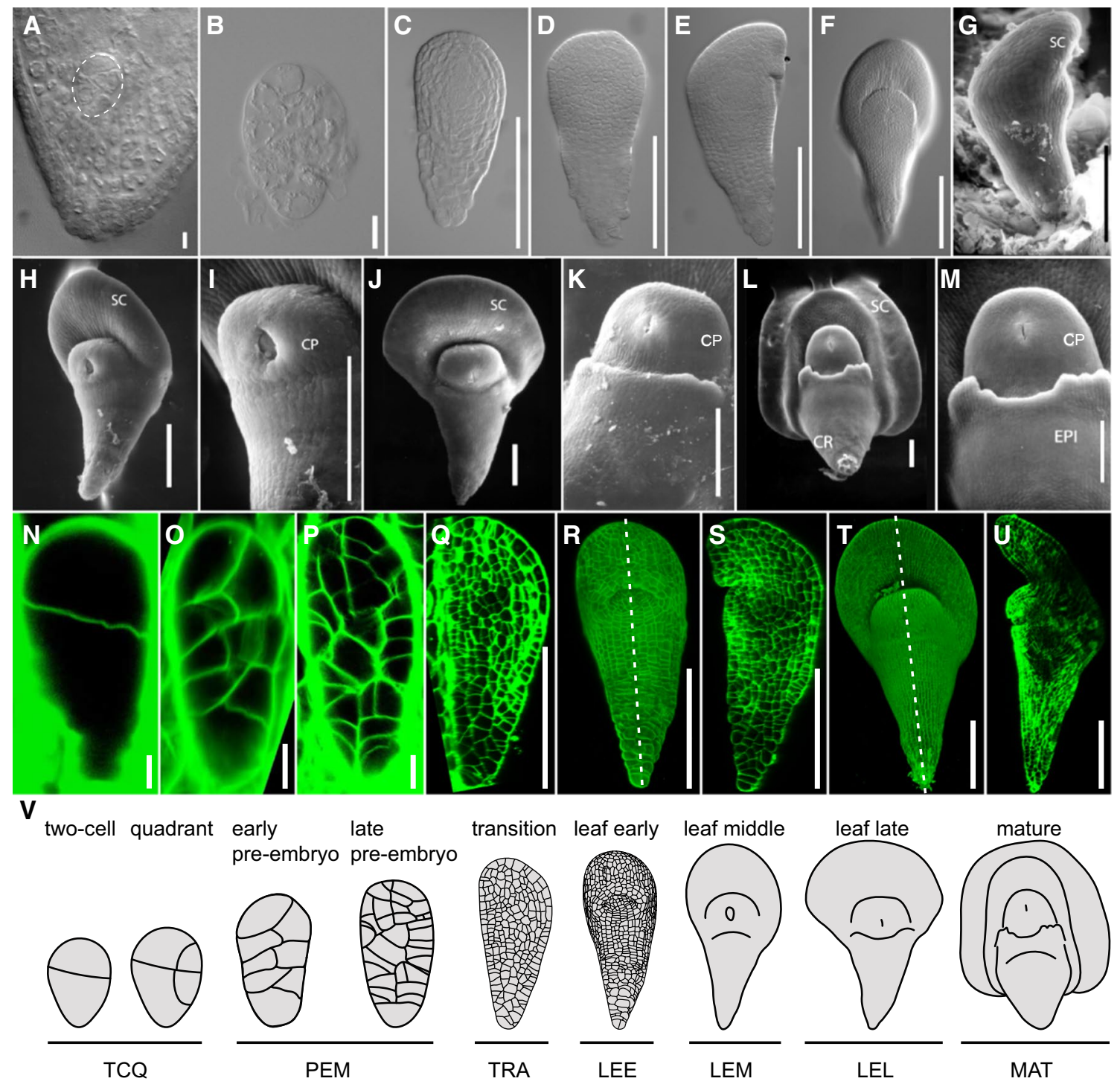

Fig. 1 Development of Brachypodium embryos. Development in successive stages of Brachypodium embryos was visualized by light microscopy (A-F), scanning electron microscopy $(\mathbf{G}-\mathbf{M})$, and confocal imaging $(\mathbf{N}-\mathbf{U})$. Stages are two-cell or quadrant $(\mathbf{A}, \mathbf{N})$, proembryo $(\mathbf{B}, \mathbf{O}, \mathbf{P})$, transition $(\mathbf{C}, \mathbf{D}, \mathbf{Q})$, leaf early $(\mathbf{E}-\mathbf{G}, \mathbf{R}, \mathbf{S})$, leaf middle $(\mathbf{H}, \mathbf{I}, \mathbf{T}, \mathbf{U})$, leaf late $(\mathbf{J}, \mathbf{K})$, and mature $(\mathbf{L}, \mathbf{M}) . \mathbf{S}, \mathbf{U}$ are

anatomically recognizable until the leaf late stage (Fig. 1U). The scutellum was developed into a shield-shape, and a bulging coleoptile was clearly observed in the leaf middle stage (Fig. 1T, U, V). At the same time, differentiation of epiblast cells occurred and further developed in the two subsequent stages, the leaf late and mature stages (Fig. 1J-M, V). Thus, while clear organs and structures are anatomically visible at the LEE, LEM, LEL, and MAT stages, no clear landmarks of patterning can be observed prior to this. Yet, the first divisions of the zygote are regular, which suggests the potential existence of an early pattern formation process.

optical section along the white dashed lines in $(\mathbf{R}, \mathbf{T})$. Embryos in $(\mathbf{A}$, $\mathbf{N}-\mathbf{P}$ ) are inside seeds, while all others were removed from the seed. V Full series of developmental stages and nomenclature. Scale bars: $5 \mu \mathrm{m}$ in $(\mathbf{A}, \mathbf{N}), 10 \mu \mathrm{m}$ in $(\mathbf{B}, \mathbf{O}, \mathbf{P}), 50 \mu \mathrm{m}$ in $(\mathbf{I}, \mathbf{K}, \mathbf{M})$ and $100 \mu \mathrm{m}$ in $(\mathbf{C}-\mathbf{H}, \mathbf{J}, \mathbf{L}, \mathbf{Q}-\mathbf{U})$. $S C$ scutellum, $C P$ coleoptile, $C R$ coleorhiza, EPI epiblast

\section{A reference transcriptome of the developing Brachypodium embryo}

To generate molecular insight in the developmental progression of Brachypodium embryogenesis, we sampled isolated embryos from the 7 stages discussed in the previous section. Considering the substantial morphological changes occurring between the leaf early stage and the leaf middle stage, we collected an additional embryo stage, with an embryo length of $180 \pm 25 \mu \mathrm{m}$ (E180), between these two stages. We also collected embryo samples with a length of $400 \pm 25 \mu \mathrm{m}$ 
Fig. 2 Division patterns in early Brachypodium embryos. Brachypodium embryos were imaged and segmented at two-cell (A), three-cell (B), four-cell $(\mathbf{C})$, pro-embryo (D) and transition stages (E) by using confocal microscopy and MorphographX. In (D) and (E), the same embryos are shown from different angles. False color scale shows the volume of cells
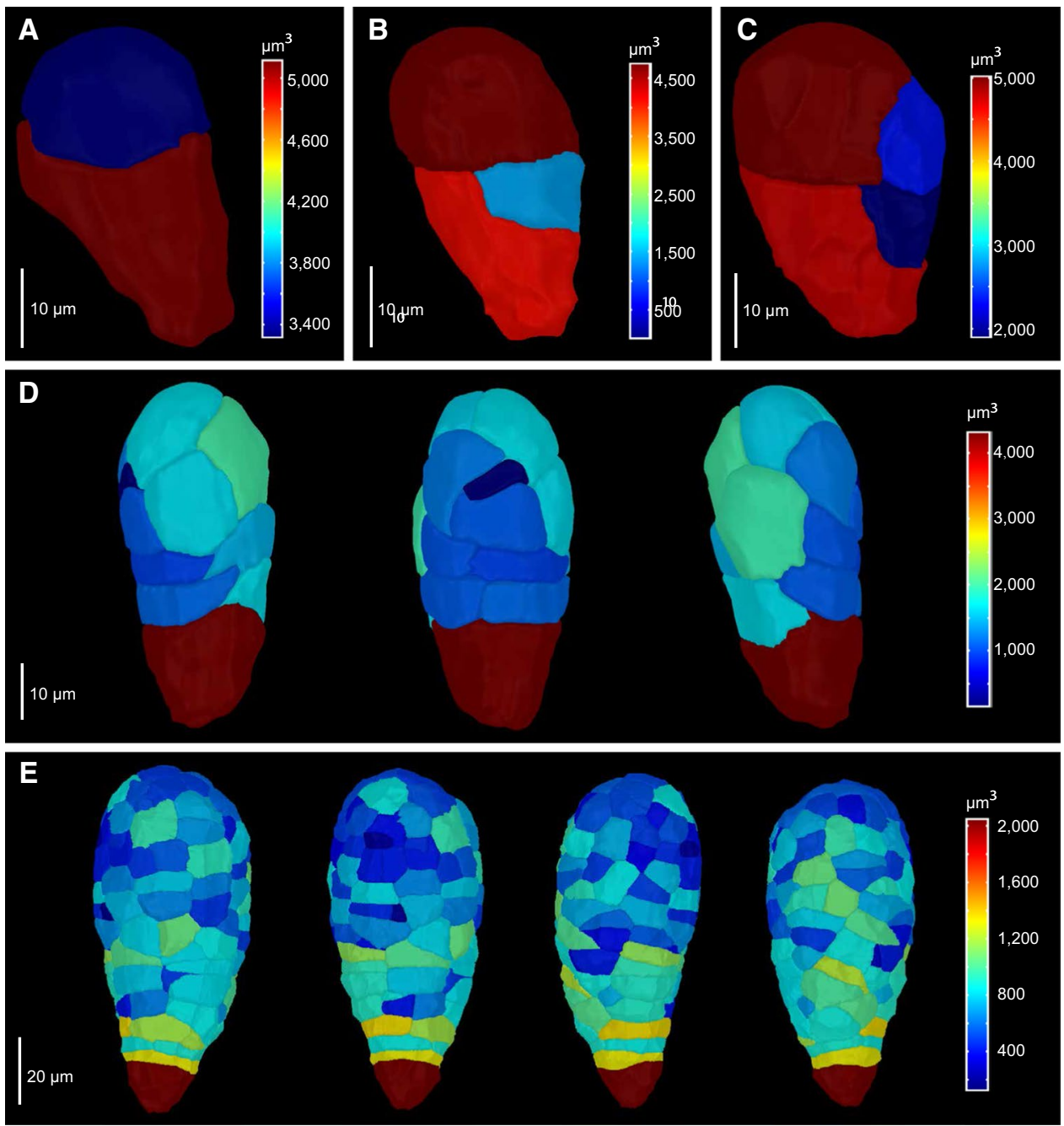

(E400), showing micro-morphological characteristics that were identical to that of embryos at the LEM stage. In addition to these nine embryo stages, we also collected three non-embryo tissues, including early endosperm (EEN), late endosperm (LEN) and seed coat (SEC), corresponding to the embryo stage of TRA, LEL and LEL, respectively. These were sampled with the aim to generate a reference transcriptome to correct for contamination with abundant endosperm and seed coat tissues during embryo isolation.

We next performed RNA-sequencing (RNA-seq) analysis on duplicates or quadruplicates of all nine embryo stages and three endosperm and seed coat samples. Among the 34,260 annotated protein-coding genes in the Brachypodium genome (The International Brachypodium Initiative 2010), $69.32 \%(23,749$ genes $)$ were expressed in at least one sample and $66.99 \%(22,951$ genes $)$ were differentially expressed (FDR <0.05) between at least two different samples. Hierarchal clustering of the significant changes in gene expression across all samples revealed a progressive transcriptomic shift during $B$. distachyon embryo development and an obvious tissue-specific transcriptome profile between embryo and non-embryo tissues (Supplementary Figs. S1 and S2).

A major concern with manual dissection and sampling of early embryos from the much larger seed coat and dense endosperm is the contamination with non-embryo tissues. Studies in Arabidopsis showed that such contamination may confound embryo transcriptome profiling and lead to contentious inferences (Schon and Nodine 2017). Thus, despite making great efforts to avoid contamination during embryo sampling (see Methods), we quantified the expression of some well-known tissue-specific genes across all samples to address the degree of non-embryo tissue contamination. Glutelin is a well-known seed storage protein, which has an endosperm-specific expression pattern in rice (Takaiwa et al. 1996). In the B. distachyon genome, glutelin is encoded by seven genes, all of which are specifically expressed in endosperm (Supplementary Fig. S3). Cellulose biosynthesis plays a very important role during seed coat development, particularly in secondary cell wall reinforcement and mucilage attachment (Griffiths and North 2017; Mendu 
et al. 2011). Seven cellulose synthases are expressed during Brachypodium embryogenesis, four of which are highly expressed in seed coat (Supplementary Fig. S4). In addition, studies in Arabidopsis (Kunieda et al. 2013), soybean (Gijzen et al. 1993) and prickly sida (Egley et al. 1983) showed that some peroxidases, heme-containing proteins, accumulated in and contribute to seed coat development. Among the 154 peroxidases in the Brachypodium genome, 82 are expressed during Brachypodium embryogenesis and 17 are highly expressed in seed coat (Supplementary Fig. S5). Lastly, we examined the expression of the closest Brachypodium homologs of the Arabidopsis ZHOUPI (Yang et al. 2008) and Wheat $N A C 019$ (Gao et al. 2021) genes, both of which are specifically expressed in endosperm. The Brachypodium homologs are likewise strongly expressed in the endosperm sample (Supplementary Fig. S6). None of these inferred endosperm- or seed coat-enriched transcripts was found to be expressed at appreciable levels in the isolated embryos (Supplementary Figs. S3-S6), and indeed, principal component analysis clearly separates the endosperm and seed coat samples from all embryo samples (Supplementary Fig. S2). We thus conclude that there is minimal contamination in the embryo samples.

\section{Cross-species genomic conservation of embryo development}

Morphological patterns of embryo development are very different between monocotyledonous and dicotyledonous plants, and it is an entirely open question whether the progression of developmental events and biological processes follows similar or different trajectories between these divergent groups. To address this question, we used our highquality temporal transcriptome series for a comparison with datasets derived from the $\operatorname{dicot} A$. thaliana, for which several embryo transcriptomes have been reported.

We combined two datasets (Hofmann et al. 2019; Nodine and Bartel 2012) to cover both early embryogenesis and late embryogenesis at high temporal resolution. A total of 19,893 genes $(41.14 \%$ of all annotated genes) could be detected in embryo samples, and 17,314 genes are differentially expressed (FDR $<0.05)$ between any two of the developmental stages. A principal component analysis (PCA) showed that the first two PCs cumulatively explained $69.23 \%$ of the total variance, and all samples were separated according to their developmental stage (Fig. 3B). We generated a comparable PCA plot for our Brachypodium dataset. The PC1 and 2 represented $73.26 \%$ of the total variance and stratified all samples in a successive, but distinct developmental trajectory (Fig. 3A). The PCA indicates that although great differences exist in the external embryo development of $A$. thaliana and $B$. distachyon, both of their embryogenesis trajectories appear as a gradual development not only at the morphological level but also at the global gene expression level.

To relate developmental progression between Arabidopsis and Brachypodium embryogenesis, we calculated a pseudotime metric (Leiboff and Hake 2019), namely developmental time units (DTUs), to reconstruct the molecular ontogenies for each species using the expression trajectory information in their respective PCA plots (Fig. 3C, D). Then, to determine whether individual Brachypodium embryo developmental stages can be matched with comparable Arabidopsis stages, we performed a hierarchical clustering based on the relative expression data of orthologous genes. This analysis showed that transcriptomes of classes of embryo stages are more similar between species than with different stage classes within species. While the embryo stages between these two species cannot be directly matched, they can be roughly classified into three distinct developmental phases, i.e., early, middle, and late embryogenesis, irrespective of plant species (Fig. 3E). Within each developmental phase, tissues are clustered by species rather than stages, suggesting obvious species-specific transcriptome signatures. Furthermore, intraspecific embryo transcriptome comparisons also reveal that early and late embryogenesis are more conserved between species, and these are separated by a phase of dramatic differences in gene expression (Fig. 3F).

\section{Conserved and diverged functions during embryo stages across angiosperms}

To study the deep phase conservation and divergence in terms of gene expression between Arabidopsis and Brachypodium embryo development, we mapped the expression profile of each gene to one of the developmental phases, i.e., early, middle and late embryogenesis, to identify phasespecific genes of which expression was restricted to one of these three developmental phases (Fig. 4A-D, I-L). We next compared the patterns of orthologous genes between the two species and found that phase specificity of orthologs was much more prominent in early and late embryogenesis than in middle embryogenesis (Fig. 4E-H), consistent with the results of the interspecific transcriptome comparison (Fig. 3F). Gene Ontology (GO) enrichment analysis indicates that the early phase is enriched for ribosome, translation and DNA replication which are associated with cell growth and proliferation (Fig. 4M, N and Supplementary Fig. S7). In contrast, the late phase is enriched for various enzyme activities, transporters and signaling which reflect a cell type-specific status, suggesting that this phase is characterized by genes expressed in differentiated and specialized cells (Fig. 4M, N and Supplementary Fig. S7). Compared to the early and late phases, the transcriptomes of middle embryogenesis of Arabidopsis and Brachypodium are less 


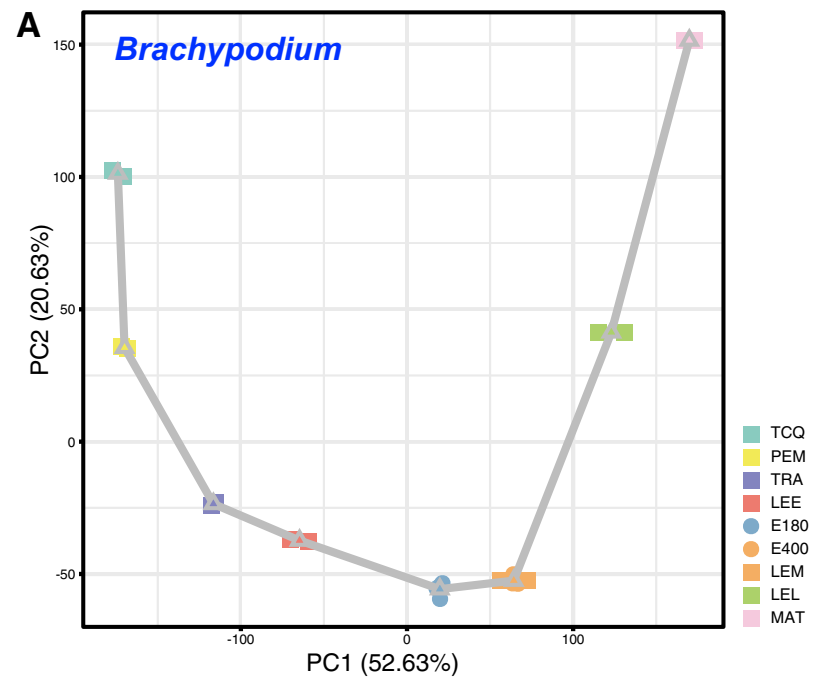

C

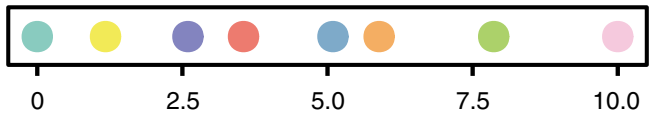

E

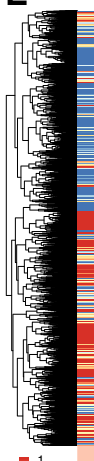

MG MAT LEL E180 LEM BC

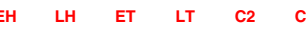

C8

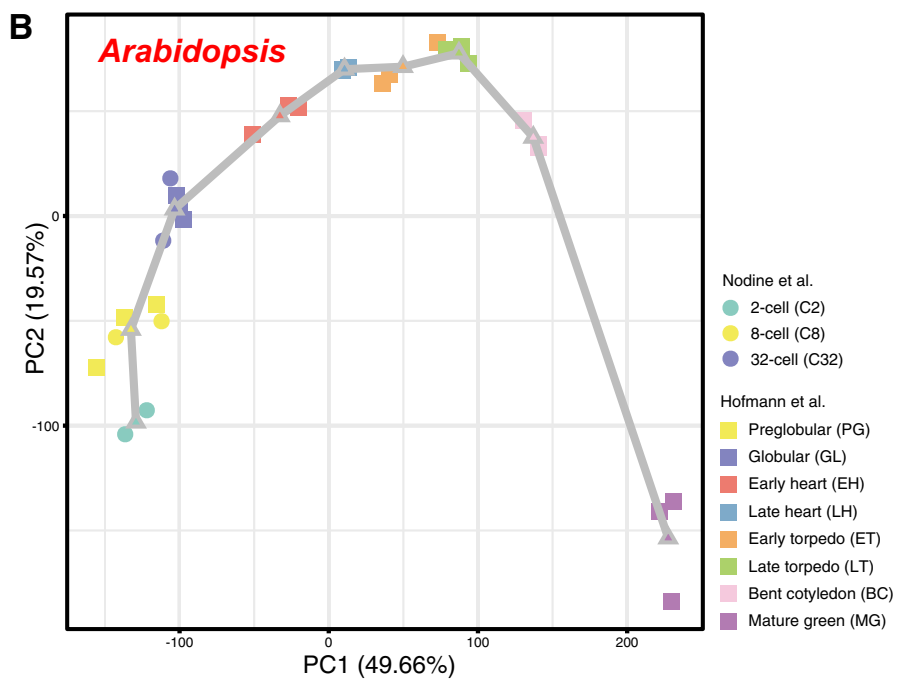

D

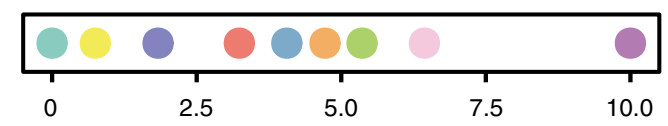

$\mathbf{F}$

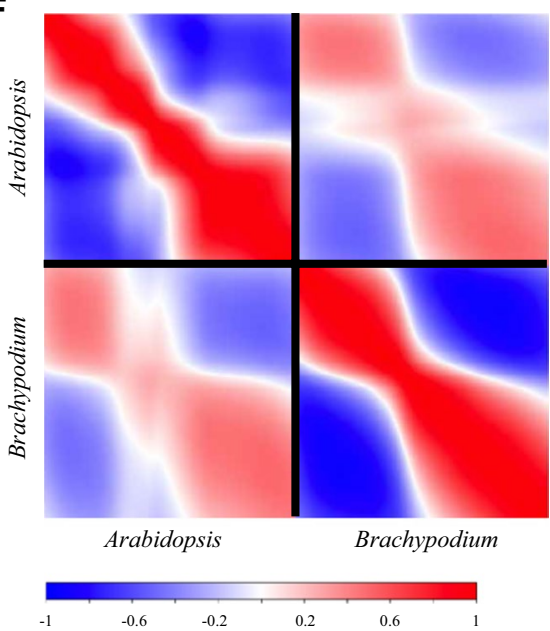

Fig. 3 Transcriptional dynamics of $B$. distachyon and A. thaliana embryogenesis. All of the differentially expressed genes were used in the PCA plots to stratify embryonic transcriptomes across stages in Brachypodium (A) and Arabidopsis (B). Each developmental stage was represented using the centroid location in each PCA plot and adjacent centroids were linked using straight lines, forming an expression trajectory. Then, the rank and distance along the developmental trajectory were used to calculate a developmental time units (DTU) value, scaled from 0.0 to 10.0 , as a pseudotime metric in each species $(\mathbf{C}, \mathbf{D})$. E The hierarchical clustering of the relative expres-

correlated, with only few overlapped phase-specific orthologous genes (Figs. 3F and 4G). Those limited gene sets are enriched in chloroplast-related GO functional terms (Supplementary Fig. S8). Thus, while the mid-embryo development phase is morphologically and transcriptionally divergent between Arabidopsis and Brachypodium, a common characteristic is active chloroplast development. sion data of orthologous genes between Brachypodium and Arabidopsis shows broad clustering according to embryogenesis phase (early/ middle/late). Stage abbreviations are the same as in the key to (A) and (B). F The intra- and interspecific comparisons of embryonic developmental transcriptomes between Brachypodium and Arabidopsis. The successive stages of embryogenesis (DTU plots from panels $\mathbf{C}$ and $\mathbf{D})$ are plotted on both axes of each plot. Species are indicated on each axis. Pearson correlation: positive, red; no correlation, white; negative, blue

Next, we asked if the middle phase between the early and late embryogenesis is enriched in specific gene families in a species-dependent manner. To achieve this, we first downloaded a collection of gene families of Arabidopsis from TAIR (www.arabidopsis.org) and identified homologs in Brachypodium by a best blast hit approach. Gene set enrichment analysis (GSEA) indicates a similar result with previous GO enrichment for early and late phases (Supplementary 


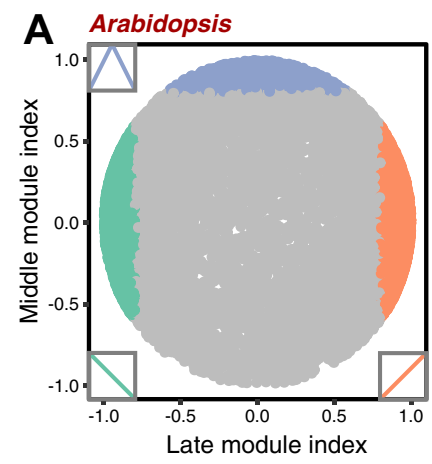

E
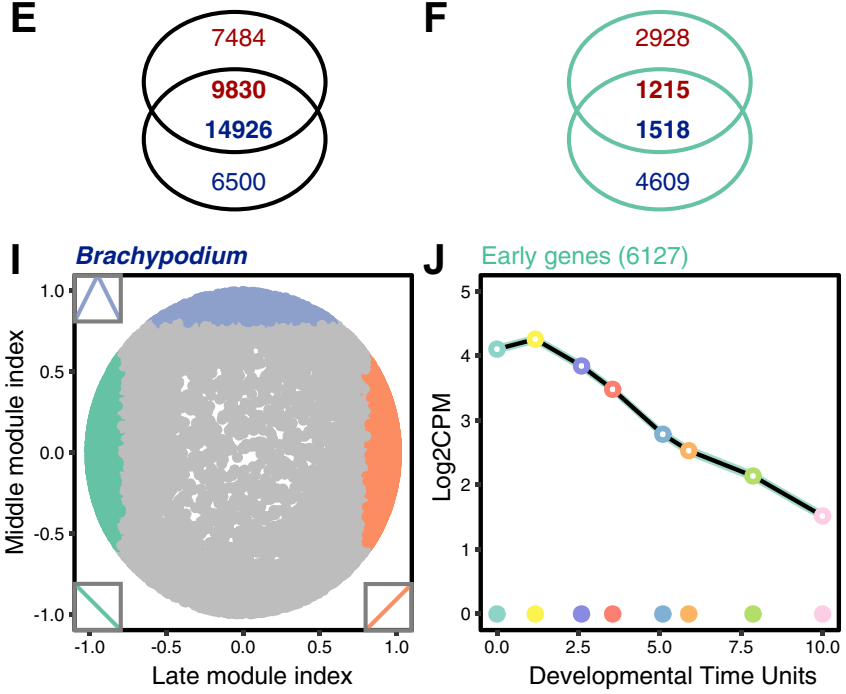

M Arabidopsis

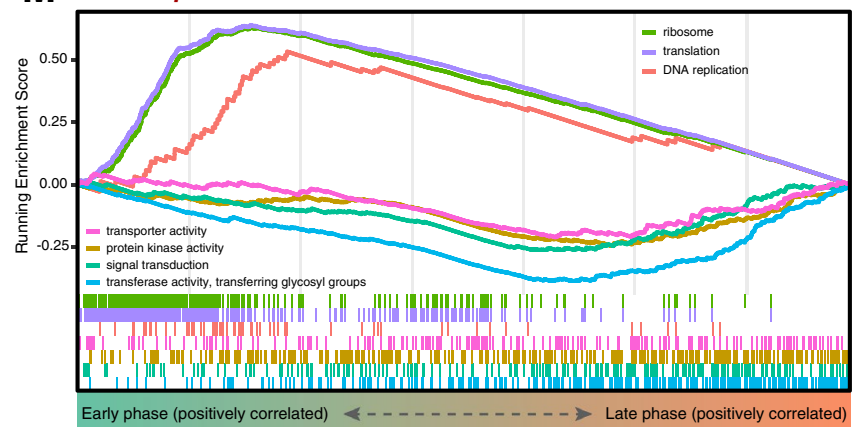

Fig. 4 Phase-specific gene expression and functional enrichment. (A, I) Landscapes show the correlations between gene expression profiles and two hypothetical perfect modules: middle ( $y$ axis) and late $(x$ axis) modules, during embryogenesis in Arabidopsis (A) and Brachypodium (I). Each spot corresponds to an Arabidopsis (A) or Brachypodium (I) gene. The inset graphics show the perfect modules which are used to calculate the correlations. Three sets of phase-specific

Fig. S9). As for the middle phase, we indeed found speciesdependent enriched gene families except for two common families, zinc finger homeodomain (ZF-HD) transcription factor (TF) family and Golden2-like (G2-like) TF family (Supplementary Fig. S10). Interestingly, mid-embryogenesis
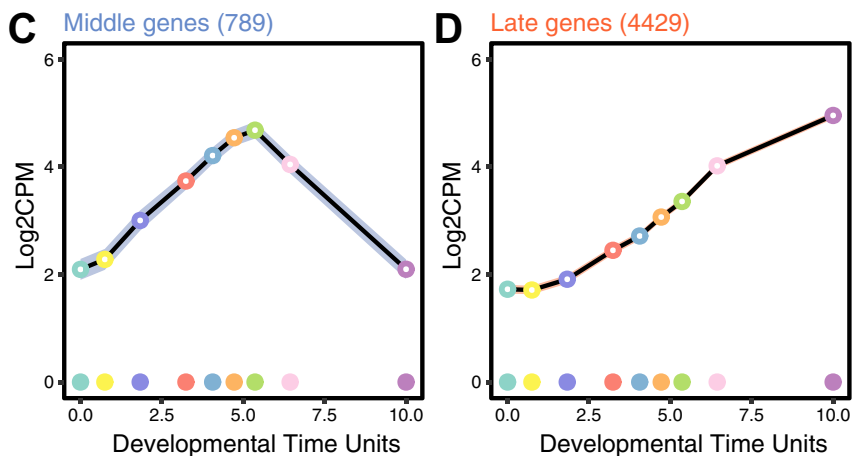

G

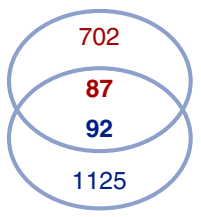

H
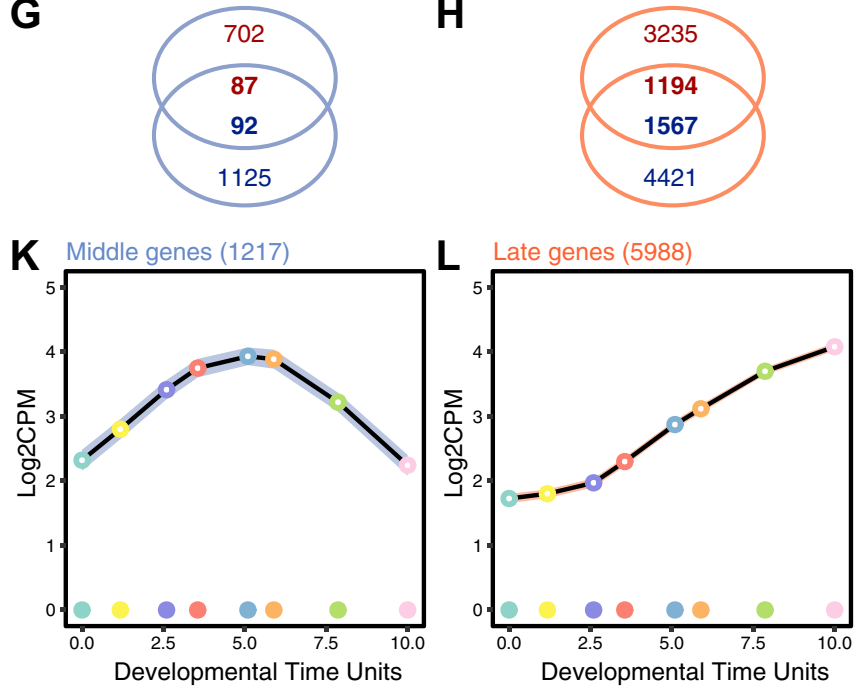

N Brachypodium

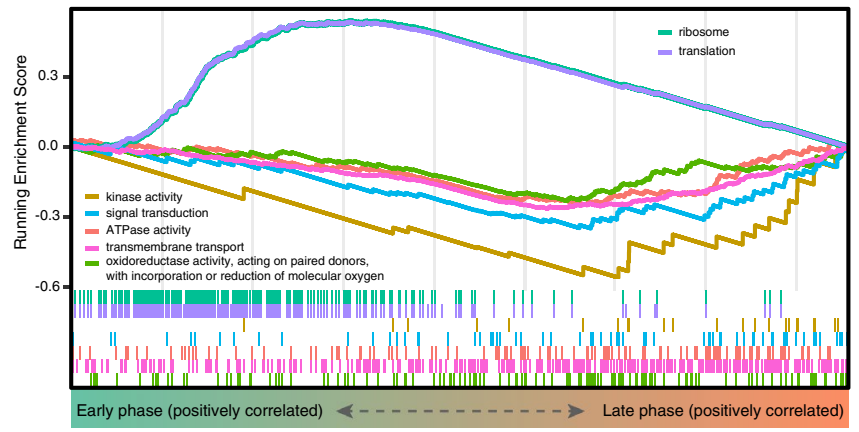

gene expression are plotted and colored according to the assigned phases, i.e., early (cyan), middle (blue) and late embryogenesis (orange) in Arabidopsis (B-D) and Brachypodium $(\mathbf{J}-\mathbf{L})$. The number of overlapped orthologous genes for each set is also indicated $(\mathbf{E}-\mathbf{H})$. Gene set enrichment analysis (GSEA) shows the gene sets which are highly correlated with early or late phases in Arabidopsis (M) and Brachypodium $(\mathbf{N})$

in Arabidopsis is enriched for two gene families, $A S Y M$ METRIC LEAVES2 (AS2) and LATERAL ORGAN BOUNDARIES $(L O B)$, that function in the organ asymmetry and boundary formation (Iwakawa et al. 2002; Semiarti et al. 2001; Shuai et al. 2002), respectively (Supplementary Fig. 
S10C). Mid-embryogenesis in Brachypodium is enriched for the GROWTH REGULATING FACTOR (GRF) family, which can interact with CUP-SHAPED COTYLEDON (CUC) to promote cotyledon separation in Arabidopsis (Lee et al. 2015) (Supplementary Fig. S10D). These results suggest that, while early and late phases are characterized by shared functional programs, the middle phase of embryogenesis is marked by the expression of genes involved in lineage-specific body plans.

\section{Developmental heterochrony of $B$. distachyon and $A$. thaliana embryogenesis}

Among the enriched gene sets in early and late embryogenesis, we identified several TF families. Interestingly, there was a clear difference between Arabidopsis and Brachypodium. In Brachypodium, the GL1 enhancer binding protein (GeBP), MADS-box, Homeobox, CCAAT-HAP5, Alfinlike, MYB and NAC gene families were all enriched in early and late stages (Supplementary Fig. S9B). None of these were enriched in the early phase of Arabidopsis embryogenesis (Supplementary Fig. S9A). Instead, we noticed that the Homeobox TF family was enriched in the middle-tolate phase of Arabidopsis embryo development (Supplementary Figs. S9A and S10C). Correlation of gene expression with embryo development further confirmed the expression divergence of this gene family between Arabidopsis and Brachypodium embryogenesis (Supplementary Fig. S11A, C). The same pattern was observed for the bHLH TF family, although this family was not significantly enriched in early embryogenesis in Brachypodium (Supplementary Fig. S11B, D). This finding suggests heterochronic genome-wide expression patterns between these two species for transcription factor families. Given that several members of these two TF family are key regulators of embryo patterning and tissue specification (Ito et al. 2002; Radoeva et al. 2019; Tsuda and Hake 2016), it is possible that the embryo patterning process is heterochronic between these species.

To further dissect heterochrony of early embryogenesis between Arabidopsis and Brachypodium, we initially mapped all Brachypodium genes encompassing a Homeodomain (PF00046 and PF05920) on a gene expression phasigram, which was constructed by sorting the gene expression peak along the DTUs and compared with that of Arabidopsis (Fig. 5A). We found that most Brachypodium Homeobox genes, of which the expression is restricted to early embryogenesis, have corresponding Arabidopsis homologs that are highly expressed at the middle-to-late stage of embryogenesis (Fig. 5A). Thus, most HD-containing transcription factors that are known to control Arabidopsis embryo development, for which a clear ortholog can be identified in Brachypodium, show earlier expression in Brachypodium than in Arabidopsis. Similar patterns could be observed in other TF families (Supplementary Fig. S12). Thus, if these TF homologs are functionally conserved between the two species, the homologous developmental process may occur earlier in Brachypodium.

\section{Comparable patterns of activity for developmental regulators across angiosperm embryogenesis}

After establishing that there are strong similarities in genome-wide gene expression during early and late embryogenesis and heterochronic expression of several TF families between Brachypodium and Arabidopsis, we set out to explore more systematically the correlation between temporal expression patterns of orthologous genes. We performed a dynamic time warping (DTW) expression profile alignment analysis. This analysis will compare the overall expression profiles between two time-series and calculate a DTW distance which is insensitive to local compression and stretches (Giorgino 2009). A low or high DTW distance suggests that a gene pair has a similar or dissimilar expression profile, respectively, between these two time-series datasets (Supplementary Fig. S13A). Comparative enrichment analysis shows that genes with low DTW distances are enriched in genes involved in cell proliferation, such as "DNA replication," "cell division," and "THO complex," of which the expression patterns are correlated with early embryogenesis and the genes annotated with "embryo development ending in seed dormancy" and "seed development" that are normally positively correlated with seed maturation (Supplementary Fig. S13B). These results are consistent with the results above that the fundamental processes in early and late phases during embryogenesis are relatively conserved between Arabidopsis and Brachypodium.

Although Arabidopsis and Brachypodium share a conserved developmental program regarding the fundamental processes in early embryogenesis, it is unclear how embryo patterning events, like the establishment of polar axes and the initiation and maintenance of shoot and root apical meristems (SAM and RAM), compare between species. Therefore, we surveyed DTW expression profile alignments and focused mainly on gene pairs of which the Arabidopsis homolog is well-known for its expression pattern and role in controlling embryo patterning. Surprisingly, we found that genes involved in SAM specification shared largely comparable temporal expression patterns between Arabidopsis and Brachypodium (Fig. 5B-F and Supplementary Fig. S14A, B), as well as genes for quiescent center (QC) specification (Fig. 5G, H). For the ground tissue markers, each reached their expression peaks within the early phase, but while most Arabidopsis genes retained expression afterwards, their counterparts in Brachypodium decreased dramatically afterwards (Fig. 5I-M and Supplementary Fig. S14C, D). Genes that are specifically expressed in the 

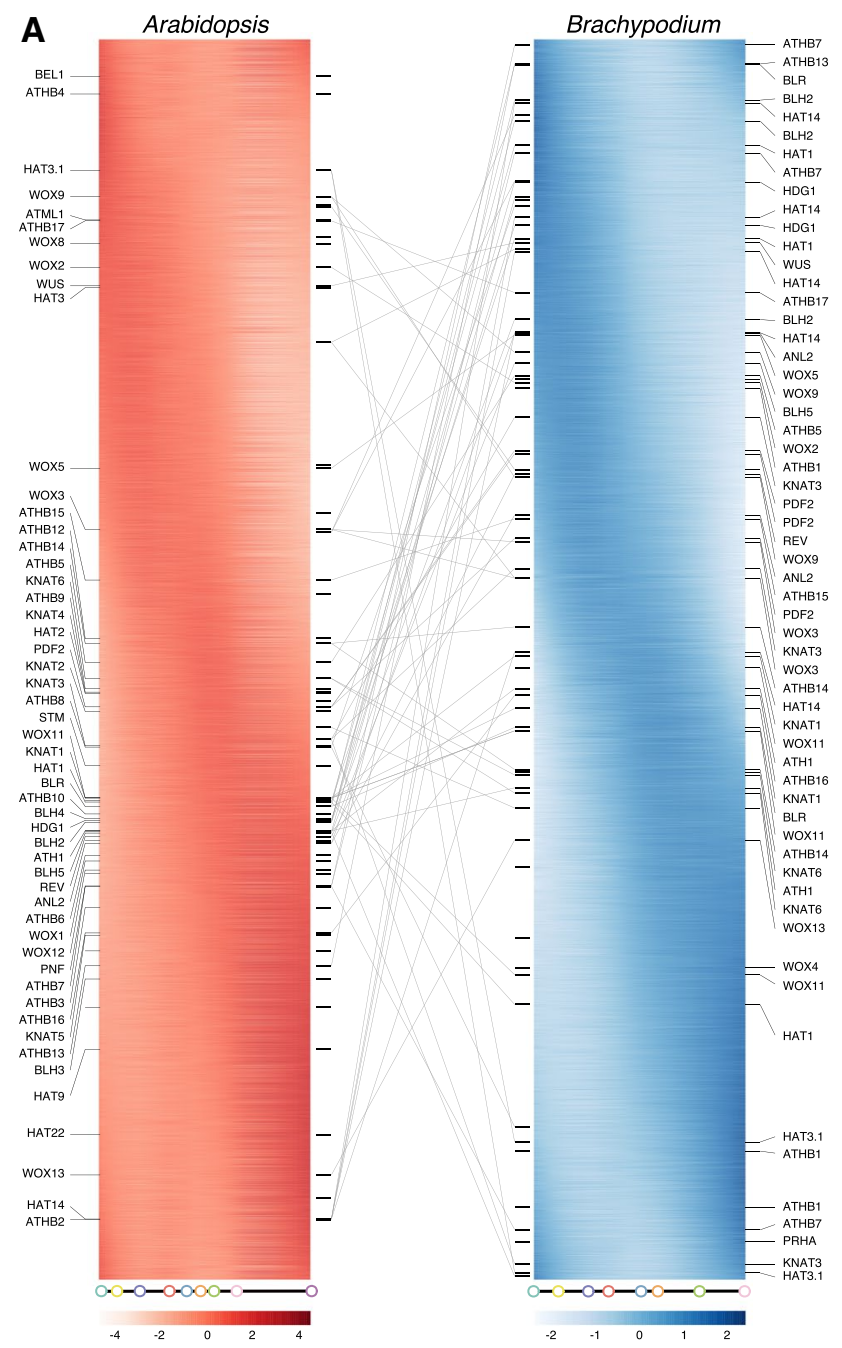

Fig. 5 Phased gene expression and dynamic timing warping expression profile alignments. (A) Arabidopsis (left, red) and Brachypodium (right, blue) homeobox genes are sorted by their expression peaks along embryonic development. All Homeobox family members that are expressed during Arabidopsis and Brachypodium embryogenesis are mapped to the phasigrams. The lines connect homologous gene pairs of Arabidopsis and Brachypodium. (B-S) DTW alignments show comparable expression patterns of cell lineage markers for shoot apical meristem (B-G; pink shading): WUS, WUSCHEL (B), STM, SHOOT MERISTEMLESS (C), KNAT1, KNOTTED-

suspensor share a similar temporal expression pattern along with the embryo development between Arabidopsis and Brachypodium (Fig. 5N, O and Supplementary Fig. S14F). As for vascular tissue specification, gene expression patterns are similar in general, however, Brachypodium genes are already relatively highly expressed at the first stage during early embryogenesis compared to their counterparts in Arabidopsis (Fig. 5P-S). We thus conclude, on the basis of comparative analysis of patterning genes, that the general progression of patterning is comparable between species. In addition, it appears that many patterning regulators are
B

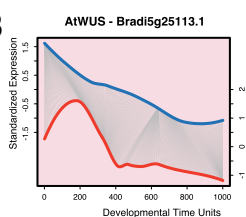

E AtZPR3 - Bradi4g42250.1

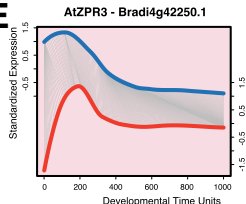

H AtPLT1 - Bradisg14960.

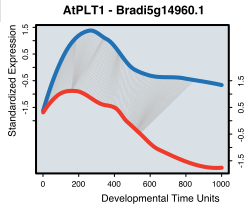

$\mathrm{K}$

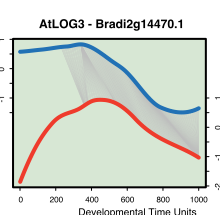

$\mathbf{N}$

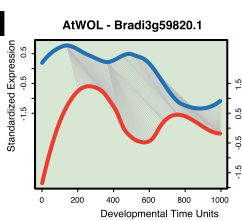

Q

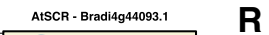

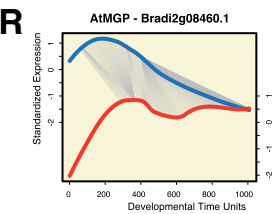

D

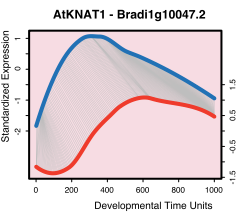

F AtCuC2 - Bradi1941712.2

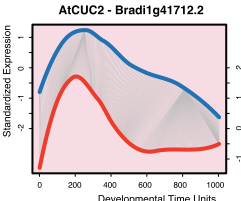

G
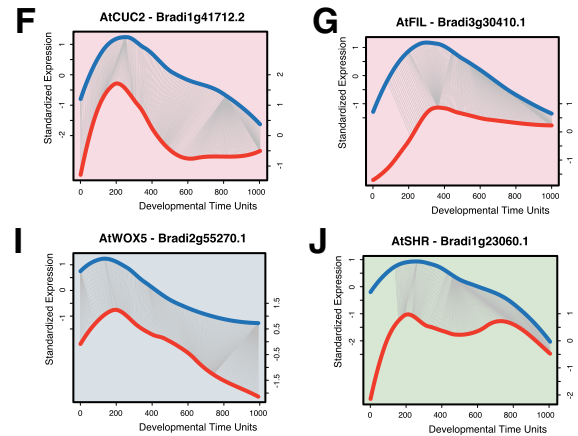

$\mathbf{L}$

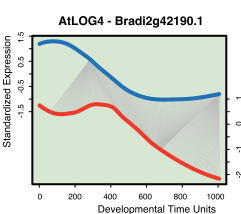

M

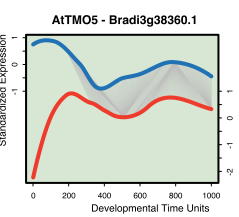

0
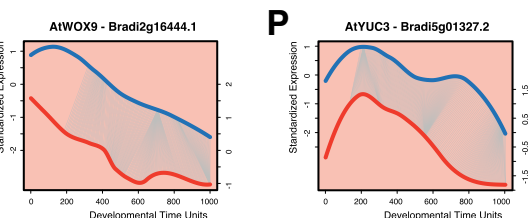

S

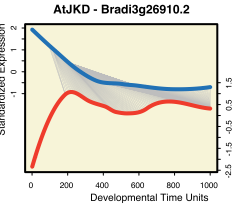

like 1 (D), ZPR3, LITTLE ZIPPER 3 (E), CUC2, CUP-SHAPED COTYLEDON 2 (F), FIL, FILAMENTOUS FLOWER (G); quiescent center (H, I; blue shading): PLT1, PLETHORA 1 (H), WOX5, WUSCHEL-RELATED HOMEOBOX 5 (I); vascular tissue (J-N; green shading): SHR, SHORT ROOT (J), LOG3, LONELY GUY 3 (K), LOG4, LONELY GUY 4 (L), TMO5, TARGET OF MONOPTEROS 5 (M), WOL, WOODEN LEG) $(\mathbf{N})$; suspensor $(\mathbf{O}, \mathbf{P}$; orange shading): WOX9, WUSCHEL-RELATED HOMEOBOX 9 (O), YUC3, YUCCA $3(\mathbf{P})$; and ground tissue $(\mathbf{Q}-\mathbf{S}$; yellow shading): SCR, SCARECROW (Q), MGP, MAGPIE (R), JKD, JACKDAW (S)

expressed early in Brachypodium, earlier than in Arabidopsis, and well before visible signs of organogenesis.

\section{Auxin activity in early Brachypodium embryogenesis}

The phytohormone auxin plays an important role in early embryogenesis in Arabidopsis (Smit and Weijers 2015). In fact, most patterning processes in the Arabidopsis embryo appear to depend on auxin response (Moller and Weijers 2009) and interference with synthesis, transport or transcriptional response each causing distinctive patterning defect 

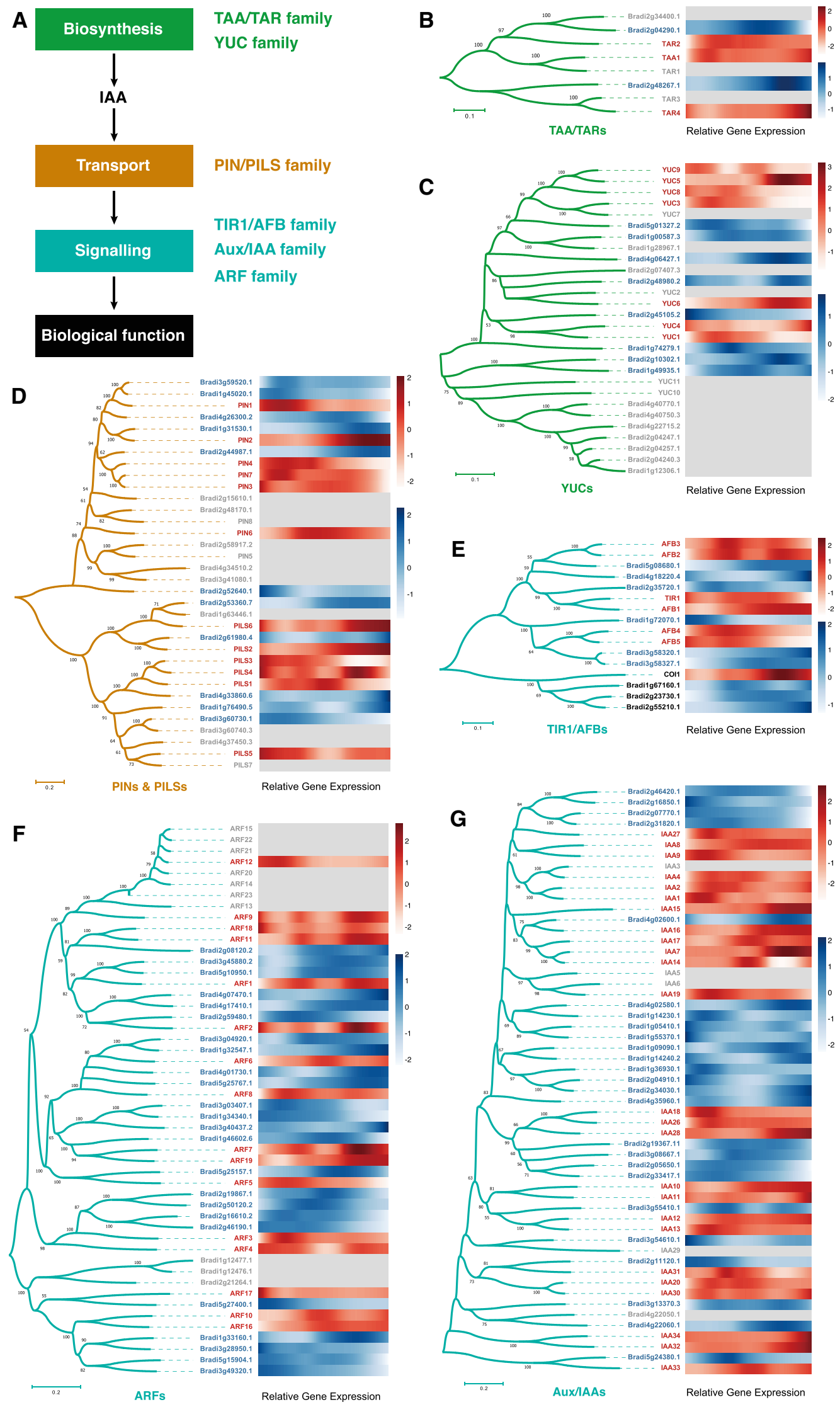
4Fig. 6 Embryonic expression of auxin-related genes in Arabidopsis and Brachypodium. (A) A schematic model of gene families that are involved in auxin biosynthesis (TAA/TAR family and YUC family), transport (PIN/PILS family) and signaling (TIR1/AFB family, Aux/ IAA family and ARF family). (B-G) Phylogenetic trees of TAA/TAR (B), YUC (C), PIN1/PILS (D), TIR1/AFB (E), ARF (F) and Aux/ IAA (G) gene families were constructed using the NJ method based on genes from Arabidopsis (red) and Brachypodium (blue). The heatmap of relative gene expression during embryogenesis is shown next to each phylogenetic tree with a white-red (lo-hi) scale for Arabidopsis and a white-blue (lo-hi) scale for Brachypodium, while genes that were not expressed were shown in gray

(Friml et al. 2003; Radoeva et al. 2019; Robert et al. 2013). It has remained unclear if a similar role for auxin is conserved in monocot embryogenesis. Analysis of the DR5 gene expression reporter in maize embryos suggests that response may not occur until mid-embryogenesis (Chen et al. 2014). Given that much of the pattern formation program is active early in Brachypodium, we explored the potential roles for auxin.

To first ask if and when auxin-related genes are expressed in Brachypodium embryogenesis and to determine their similarity to Arabidopsis orthologs, we determined temporal profiles and DTW analysis of a number of gene families: YUC and TAA/TAR biosynthesis genes, PIN transporters, TIR1/ AFB receptors, Aux/IAA repressors and ARF transcription factors (Fig. 6A). For both YUC and TAA/TAR families, we detected expression of at least one member at the earliest stages, although the peak of $B d T A A / T A R$ gene expression was later than AtTAA/TAR genes (Fig. 6B, C). Dynamics of $P I N$ gene expression in Brachypodium was similar to that in Arabidopsis and suggests early transport activity that persists during embryogenesis (Fig. 6D). TIR1/AFB receptors appear present throughout embryogenesis, and likewise, multiple members of the $B d A u x / I A A$ and $B d A R F$ families are expressed throughout embryogenesis (Fig. 6E-G). Based on these observations, the predicted capacity to synthesize, transport and respond to auxin in Brachypodium is close to that in Arabidopsis and includes the earliest stages. Only few well-characterized auxin-responsive (output) genes have been identified in the Arabidopsis embryo (Moller et al. 2017; Schlereth et al. 2010; Vaddepalli et al. 2021). While for some of these, we detected similar temporal profiles of Brachypodium orthologs (Fig. 6 and Supplementary Fig. $\mathrm{S} 15)$, its relevance is unclear.

Given the predicted ubiquitous and early auxin activity, we explored the ability of embryos to transport and respond to auxin. To this end, we analyzed the localization of transporters PIN1a, PIN1b and SoPIN1 (PINla-Citrine, PINbCitrine, and SoPIN1-Citrine), as well as expression of the synthetic auxin-responsive promoter DR5 (DR5-RFP) in the developing Brachypodium embryo (O'Connor et al. 2014).

As reported by transcriptomics (Fig. 7R), we could indeed detect each PIN protein early during embryogenesis. PIN1a and PIN1b had similar expression patterns (Fig. 7A-M), both were detected in the inner cells of pro-embryo, but PIN1a was more concentrated in the presumed vascular area (Fig. 7A-G), whereas PIN1b was expressed in a broader domain than that of PIN1a (Fig. 7H-M). Furthermore, PIN1a was expressed at a very early stage, which was temporally consistent with the RNA-seq profiling (Fig. 7R), showing a spatially polarized localization towards the one specific cell side (Fig. 7A). Interestingly, soPIN1 had a different expression pattern, which was specifically expressed at the apical domain in the transition stage (Fig. $7 \mathrm{~N}, \mathrm{O}$ ) and predominantly expressed at the apical domain afterwards (Fig. 7P, Q). Thus, based on polar localization of PIN proteins, the early Brachypodium embryo likely has the capacity to directionally transport auxin.

Despite early expression of auxin biosynthesis, transport and response components (Fig. 6) and PIN localization, we did not observe DR5-RFP activity until the transition stage (Fig. 7S-V). At earlier stages, we did detect activity in surrounding maternal tissues (Fig. 7S), similar to patterns observed in Arabidopsis and maize (Chen et al. 2014; Robert et al. 2018). From transition stage onward, DR5-RFP was found to be expressed in the root tip, the vasculature and the tip of the scutellum (Fig. 7T). The auxin signaling at the apical of scutellum was expanded to the entire edge of scutellum in the leaf middle stage (Fig. 7U, V). At these stages, PIN patterns and DR5-RFP activity were in good agreement.

In conclusion, from transition stage onward, the Brachypodium embryo is marked by prominent auxin transport and response, which align well with the establishment of vascular tissue and root. At earlier stages, transcriptome analysis predicts extensive auxin activity, but the only available reporter for response could not confirm this activity.

\section{Discussion}

Many fundamental questions related to embryo development in monocots, and by extension, to the conserved and divergent properties between monocots and dicots, have remained unanswered. Here, we have investigated the cellular patterns and gene expression of Brachypodium embryogenesis to address such questions. First, we analyzed the pattern of divisions in detail to address the question of whether there is regularity in early divisions. In Arabidopsis, most divisions in the embryo are essentially invariant, leading to near-complete predictability of the pattern formation process (Mansfield and Briarty 1991). However, this regularity is not shared with many other plants, and descriptions of maize and rice embryogenesis suggest that monocot embryos do not follow a strictly defined pattern of divisions (Chen et al. 2014; Itoh et al. 2005). Through segmentation of cells in early 

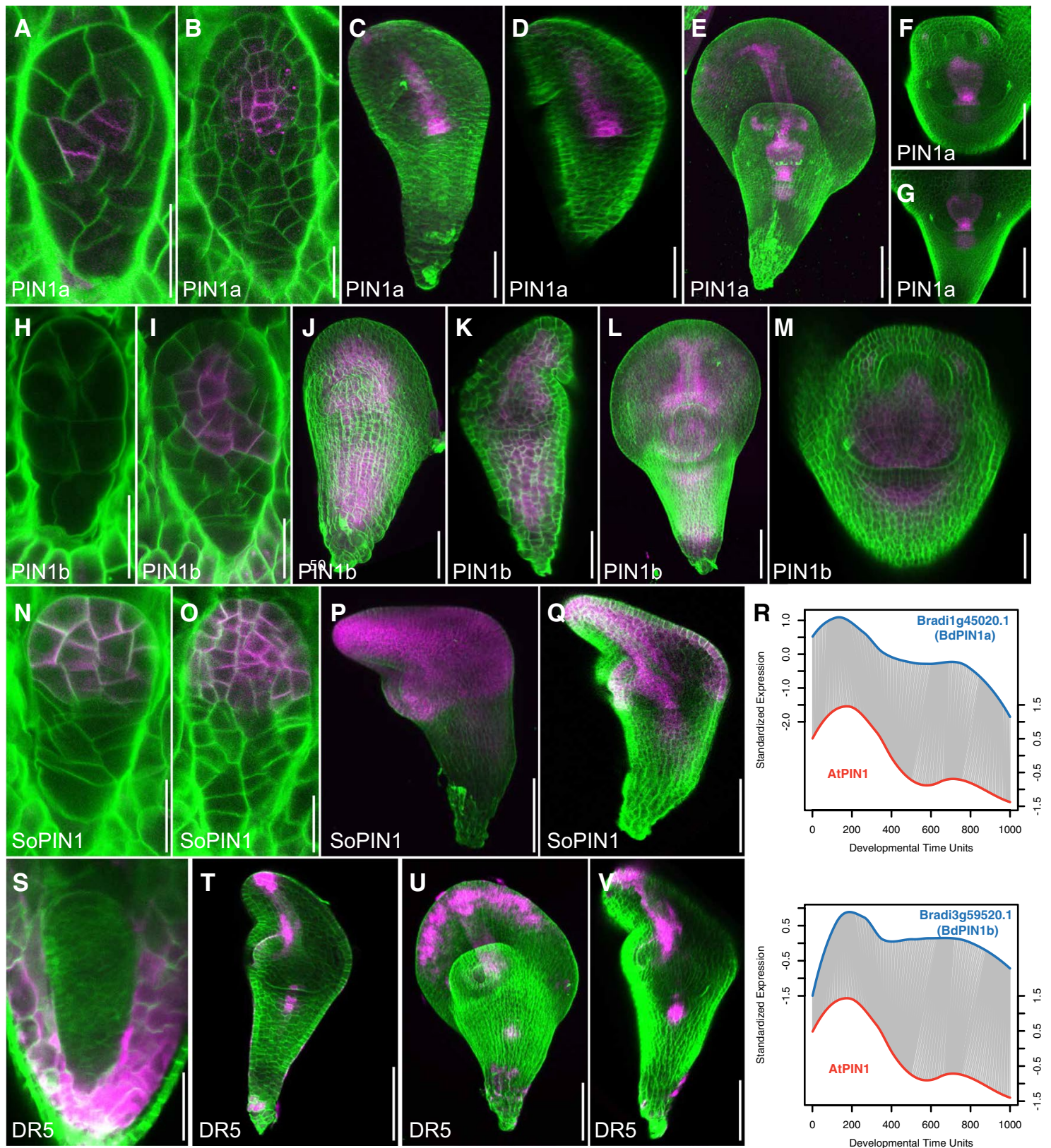

Fig. 7 Auxin transport and response during Brachypodium embryogenesis. (A-Q) Localization of PIN1a-Citrine (A-G), PINb-Citrine $(\mathbf{H}-\mathbf{M})$ and SoPIN1-Citrine (N-Q) during Brachypodium embryogenesis. Magenta color shows PIN protein localization and green color is Renaissance cell wall staining. (R) DTW alignments show comparable temporal expression patterns of PIN1 and SoPIN. (S-V) Expression of DR5-GFP (magenta) in Brachypodium embryos. Green color is renaissance cell wall staining. Scale bars: $20 \mu \mathrm{m}$ in $(\mathbf{A}, \mathbf{B}, \mathbf{H}$, $\mathbf{I}, \mathbf{N}, \mathbf{O}), 50 \mu \mathrm{m}$ in $(\mathbf{C}, \mathbf{D}, \mathbf{J}, \mathbf{K}, \mathbf{P}, \mathbf{Q}, \mathbf{S})$, and $100 \mu \mathrm{m}$ in $(\mathbf{E}-\mathbf{G}, \mathbf{L}, \mathbf{M}$, $\mathbf{T}-\mathbf{V})$ embryos, we find that the first two rounds of cell division in the Brachypodium embryo show regularity in both being asymmetric. These two division rounds set apart two small cells from which the central embryo axis likely develops. Later divisions are not strictly controlled, but do further elaborate the early pattern. This finding suggests that there may in fact be a very early pattern formation step in which a domain with distinct developmental fate is defined. Where intuitively, based on morphology, one would perhaps expect pattern formation to be delayed in Brachypodium. Compared to Arabidopsis, it may in fact commence early.

Through developing a transcriptome resource of the successive embryo stages, we were able to address this question 
more directly. Given the long time that has passed since the lineages giving rise to Arabidopsis and Brachypodium split from their last common ancestor, there is no simple orthology relationship between Arabidopsis and Brachypodium genes, which makes it difficult to infer developmental progression using the expression pattern of known Arabidopsis regulators. We therefore first asked if the global progression of embryogenesis is conserved between species. We found that it is and that particularly the early and late phases of embryogenesis share common transcriptomic features. This suggests, perhaps unsurprisingly, that the physiological and functional processes that mark early and late embryogenesis are conserved across angiosperms. At the same time, the middle phase of embryogenesis showed little global similarity between species, which correlates with the vastly different morphologies observed. Nonetheless, when exploring the temporal expression profiles of the most similar among co-orthologs and gene family members of known Arabidopsis developmental markers and regulators, we did find that their patterns were in fact similar. This is in itself interesting, because it suggests that the timing of expression of developmental genes can be uncoupled from their evidently different spatial patterns that dictate species-specific morphologies. At the same time, this finding suggests that regulation of development may share substantial parts of regulatory networks across angiosperms.

Our finding that early and late embryogenesis are more alike at transcriptome level between two divergent angiosperm species than middle embryogenesis is in interesting contrast to the transcriptomic "hourglass" model that was proposed based on analysis of Arabidopsis embryo transcriptomes (Quint et al. 2012). In this analysis, the transcriptomes of various stages of Arabidopsis embryos were queried for the evolutionary age of the genes that are expressed. This led to a pattern where during mid-embryogenesis (heart-torpedo stage), transcriptomes are dominated by "old" or less divergent genes. To compute transcriptome age, the presence/ absence of homologs across a phylogenetic tree was scored, with genes having homologs across more divergent organism being binned in "older" phylostrata. In contrast, we did not consider transcriptome "age," but based our analysis of divergence and similarity on the expression patterns of closest homologs between Arabidopsis and Brachypodium based on best BLAST hits. Therefore, our study identifies similarity patterns irrespective of transcriptome "age." It is well possible that many of the genes with shared patterns across the two species are in fact angiosperm-specific and thus part of a "young" phylostratum. A deeper analysis of transcriptome age across these two angiosperm embryo series will be informative in enriching the hourglass model.

A notable observation was that there appears to be a clear heterochrony of embryo development between
Arabidopsis and Brachypodium. Based on the expression patterns of large transcription factor families, and other developmental regulators, patterning may occur earlier in Brachypodium than in Arabidopsis. This is counterintuitive given the lack of early discernable landmarks of embryo patterning in Brachypodium, but does align with the finding that the first divisions in the Brachypodium embryo are highly regular. We predict based on these findings that the embryo patterning process occurs early in Brachypodium and expect that future investigation of expression patterns of developmental regulators will shed light on the spatiotemporal establishment of cell fates and their regulation.

In Arabidopsis, one such regulator is the plant hormone auxin. This small molecule has been implicated in many aspects of embryo development (Moller and Weijers 2009; Smit and Weijers 2015), but based on analysis of the auxin response reporter DR5 in maize, it is questionable if there is active signaling in early embryonic stages (Chen et al. 2014). As was found in the earliest steps of maize embryogenesis (Chen et al. 2014), we also find expression of all components in auxin biology throughout embryogenesis, but the DR5 reporter was likewise not active until transition stage. Active signaling can only be inferred from the expression of genes that are activated by auxin response, of which very few are known, even in Arabidopsis. Thus, also in Brachypodium, it remains an open question whether early embryonic stages feature auxin response, and if so, whether it contributes to patterning. We do find that the PIN1 proteins, i.e., BdPIN1a, BdPIN1b and BdSoPIN1 (O'Connor et al. 2014), are expressed and polarized early during Brachypodium embryogenesis. This suggests that at the very least, all hallmarks for active auxin homeostasis, transport and response are there, which makes it rather unlikely that this system is not used during early embryogenesis. One notable difference between Arabidopsis and Brachypodium though is the absence of the PIN3/4/7 clade in the latter. These PIN proteins are prominently active in the early Arabidopsis embryo (Friml et al. 2003 and may endow the dicot embryo with unique regulatory abilities. The reporter used to measure auxin response, DR5, is a direct repeat of a medium-affinity binding site for ARF proteins (Boer et al. 2014; Ulmasov et al. 1997), and it is possible, likely even, that this element topology only reports part of the auxin response system. The use of new, high-affinity binding sites with different topologies (Liao et al. 2015) may help to address the question of whether auxin response contributes to early embryo development.

Lastly, our study provides an expression resource for probing genes activity during Brachypodium embryogenesis. After maize (Yi et al. 2019) and wheat (Xiang et al. 2019), it is one of the few such resources in monocots, particularly in a non-domesticated species. We expect that 
deeper analysis will help provide insights into the unique biology of the monocot embryo.

\section{Materials and methods}

\section{Plant materials}

B. distachyon $\mathrm{Bd} 21$ plants were grown in growth chambers under long-day conditions of $16 \mathrm{~h}$ of light, $22{ }^{\circ} \mathrm{C}$ and $8 \mathrm{~h}$ of dark, $20^{\circ} \mathrm{C}$, with light intensity of $100-120 \mu \mathrm{mol}^{-2} \mathrm{~s}^{-1}$ (Philips high-output F54T5/835-841 bulbs) for the whole life cycle. Spikelets were emasculated and pollinated at the flowering stage to ensure sufficient and developmentally coordinated grain production for embryo isolation.

\section{Microscopy}

Embryos were cleared in chloral hydrate solution $(8: 1: 2$, chloral hydrate/glycerol/water, w/v/v) and viewed with a Leica DMR compound microscope with Nomarski optics. Images were captured using a MagnaFire camera (Optronics) and were edited in Adobe Photoshop CS (Xiang et al. 2011b). Scanning electron microscopy was performed as described previously (Venglat et al. 2011; Xiang et al. 2019) for isolated embryos. For the Brachypodium grain, longitudinal hand sections through the grain were made prior to submerging the samples in $25 \mathrm{mM}$ PIPES, $\mathrm{pH}$ 7.0, containing $2 \%(\mathrm{v} / \mathrm{v})$ glutaraldehyde for $2 \mathrm{~h}$. After several washes, the samples were fixed in $2 \% \mathrm{OsO}_{4}$ in $25 \mathrm{mM}$ PIPES for $2 \mathrm{~h}$, washed and dehydrated in ethanol $(30,50,70,95$ and three $100 \%$ exchanges).

After sample dehydration, substitution to amyl acetate was performed with increasing ratios of amyl acetate to ethanol (spanning 1:3 parts [v/v], 1:1 [v/v], 3:1 [v/v], then two pure amyl acetate exchanges). All solvent exchanges were separated by $15 \mathrm{~min}$. Samples were critical-point dried with solvent-substituted liquid $\mathrm{CO}_{2}$ (Polaron E3000 Series II), mounted on aluminum specimen stubs with conductive carbon glue (Ted Pella) and rotary coated with $10 \mathrm{~nm}$ of gold (Edwards S150B sputter coater). Imaging was performed with a $3-\mathrm{kV}$ accelerating voltage, $10-\mu \mathrm{A}$ current and 12.2$\mathrm{mm}$ working distance on a field emission scanning electron microscope (Hitachi SU8010).

\section{Embryo, endosperm and seed coat isolation}

Embryo isolation was performed as described for wheat and Arabidopsis previously (Xiang et al. 2011a, 2019). For endosperm isolation, the seed coat and embryo were removed from the grain and the remaining endosperm was kept for RNA isolation. For seed coat isolation, the embryo and endosperm were manually removed, and the remaining seed coat was kept. For each embryo sample in early stages of development, $\sim 50$ embryos were pooled in each biological replicate sample. For each sample in late embryo stages, a minimum of 20 embryos were pooled in each biological replicate sample. A minimum of 20 grains were used for seed coat and endosperm isolation in each biological replicate sample.

To prevent contamination of early stages of embryo mRNA (in the two-cell, pre-embryo and transition stages) by the seed coat and endosperm, we performed the isolation of embryos by puncturing a hole in the grain placed in Petri dishes containing $4.8 \%$ sucrose solution $+0.1 \%$ RNAlater (Ambion Cat\# AM7020). This approach enabled the separation of the micropylar region that houses the early stage embryo from the seed coat, and the subsequent isolation of the embryo within the micropyle separated from the endosperm cells. After one single embryo was isolated from the grain, it was transferred using a pipette to a mini petri dish, followed by gentle agitation to separate any attached debris. To ensure removal of the remaining debris, each embryo was transferred to the fresh isolation buffer in a mini petri dish for at least three rounds of sequential washing steps. All washing steps were performed on ice and inspected with a dissecting Leica microscope. After all washing steps, embryos were transferred to Eppendorf tubes on dry ice using fine glass pipettes. To test the efficacy of our isolation procedure for obtaining clean embryos (without significant contaminating endosperm or seed coat tissues), randomly selected embryos were placed on a glass cavity slide in a large droplet of water, within the confines of a well (created by a Mini PAP pen, Invitrogen Cat\# 008877) and coverslip, and inspected.

\section{RNA isolation and antisense RNA amplification}

Total RNA was extracted from embryo, endosperm and seed coat of different developmental stages following the protocol provided by the RNAqueous-Micro kit (Ambion catalog number 1927). The quantity of RNA isolated from early-stage embryos was insufficient for library preparation for RNA-seq experiments. Therefore, the mRNA from all stages was amplified and the antisense RNA (aRNA) was used for RNA-seq analysis. The mRNA amplification was conducted according to the protocol provided in the MessageAmp aRNA kit (Ambion catalog number 1750).

\section{Transcriptome analyses}

For RNA-seq profile analysis, we prepared Illumina mRNAseq libraries using the TruSeq RNA kit (version 1, rev A). Libraries were prepared with aRNA according to the 
manufacturer's instructions. For HiSeq 2000 sequencing, four libraries were pooled per sequencing lane. After quality control, read filtering and base correction for the raw read data, we used the clean read data to quantify the expression of representative gene model, the JGI v3.1 annotation of B. distachyon $\mathrm{Bd} 21$ downloaded from the Phytozome database (http://phytozome.jgi.doe.gov/), using Salmon version 0.13.0 in mapping-based mode with mapping validation (Patro et al. 2017). Read counts were used as the input for differential expression analysis using the Bioconductor package edgeR version 3.24.3 (Robinson et al. 2010). For the time-course data analysis, one-way analysis of variance (ANOVA)-like testing was performed using the glmQLFTest function in edgeR with an FDR cutoff of 0.05 .

Sample pseudotime indexing was performed as described in previous studies with some modifications (Leiboff and Hake 2019). Specifically, all of the differentially expressed genes were used to separate samples on a PCA plot for each species. Then, each developmental stage was assigned a location using the centroid value and adjacent centroids were linked using straight lines, producing an expression trajectory. Finally, the rank and distance along the developmental trajectory were used to calculate a developmental time units (DTU) value, scaled from 0.0 to 10.0 .

For temporal phased gene expression profiling (Levin et al. 2016), we standardized the logCPM profile by subtracting the mean and dividing by the standard deviation. Then, we calculated the fitted curve for each gene and interpolated the curve into 1000 points along its pseudotime metric for smooth and continuous comparisons. Next, we calculated the correlation between each gene's expression profile and two perfect modules, late and middle modules. Since the expression profile was standardized, genes formed a circle as shown in Fig. 4, with $x$ - and $y$-axis represented the correlation with the late and middle module, respectively.

To generate a phasigram (Leiboff and Hake 2019; Levin et al. 2016), we perform a PCA analysis for separating genes based on the standardized expression data. The first two components, component 1 and component 2, were used to draw the PCA plot. As the expression dataset was standardized, the genes form a circle. Then, the atan 2 function was used to order genes based on the time of expression peak, producing the phasigrams as shown in Fig. 5A.

Dynamic time warping (DTW) was performed on Arabidopsis-Brachypodium gene pairs, as determined by best blast hit approach, using the standardized expression profiles as described above and the R package dtw v1.22-3 (Giorgino 2009). GO and KEGG enrichment analyses were performed using the Bioconductor package clusterProfiler version 3.10.14 (Yu et al. 2012). Heatmaps were drawn by using the $\mathrm{R}$ package heatmap version 1.0.12.

\section{Imaging of auxin reporters}

Reporter lines were obtained from O'Connor et al. (O'Connor et al. 2014). Tissue was prepared as following: remove the lemma of the spikelet and outer layer of the ovule and excise the exposed young seed from the tip of spikelet $(200-400 \mu \mathrm{m})$. The excised tissue was then fixed and cleared according to the published method (Ursache et al. 2018). We used a SP5 upright confocal microscope (Leica) to perform all imaging analyses. Excitation wavelengths for different fluorescence markers were as following: UV laser with $405 \mathrm{~nm}$ for renaissance and $514 \mathrm{~nm}$ for mCitrine. Images were processed using LasX (Leica) or MorphGraphX software (Barbier de Reuille et al. 2015), and visualized by Photoshop (Adobe).

\section{D segmentation}

Tissue was prepared and imaged as above. The $Z$-stack depth is $0.13 \mu \mathrm{m}$. 3D segmentation was performed as described in previous studies (Barbier de Reuille et al. 2015).

Author contribution statement D.W. was the lead investigator of this research program. Z.Z., Z.H. and D.W. designed the experiments and coordinated the project. Z.Z., P.V., D.X., P.G. and R.D. performed the experimental work and collected samples. Z.H. and J.C. performed the comparative transcriptome analyses. Z.H., Z.Z. and D.W. wrote and edited most of the manuscript. All authors have read and approved the final manuscript.

Supplementary Information The online version contains supplementary material available at https://doi.org/10.1007/s00497-021-00413-4.

Acknowledgements We are grateful to Devin O'Connor for sharing the PIN and DR5 reporter lines, Christian Hardtke for sharing Bd-21 seeds and Sumanth Mutte for helpful discussions.

Funding This work was supported by Marie Curie fellowship for Zhongjuan Zhang (793058), DFG research fellowship (ZH 791/1), Wheat Flagship Program of Aquatic and Crop Resource Development Research Division of the National Research Council of Canada (RD and DX).

Availability of data and material Raw reads have been deposited in the NCBI Genome Expression Omnibus under accession number GSE168154.

Code availability Not applicable.

\section{Declarations}

Conflict of interest The authors declare no conflict of interest. 
Open Access This article is licensed under a Creative Commons Attribution 4.0 International License, which permits use, sharing, adaptation, distribution and reproduction in any medium or format, as long as you give appropriate credit to the original author(s) and the source, provide a link to the Creative Commons licence, and indicate if changes were made. The images or other third party material in this article are included in the article's Creative Commons licence, unless indicated otherwise in a credit line to the material. If material is not included in the article's Creative Commons licence and your intended use is not permitted by statutory regulation or exceeds the permitted use, you will need to obtain permission directly from the copyright holder. To view a copy of this licence, visit http://creativecommons.org/licenses/by/4.0/.

\section{References}

Agapit C, Gigon A, Girin T, Leitao L, Blouin M (2020) Split-root system optimization based on the survival, growth and development of the model Poaceae Brachypodium distachyon. Physiol Plant 168:227-236. https://doi.org/10.1111/ppl.12971

Armenta-Medina A, Gillmor CS, Gao P, Mora-Macias J, Kochian LV, Xiang D, Datla R (2021) Developmental and genomic architecture of plant embryogenesis: from model plant to crops. Plant Commun 2:100136. https://doi.org/10.1016/j.xplc.2020.100136

Barbier de Reuille P et al (2015) MorphoGraphX: a platform for quantifying morphogenesis in 4D. Elife 4:05864. https://doi.org/10. 7554/eLife.05864

Black M, Bewley JD, Halmer P (2006) The Encyclopedia of seeds. Science, technology and uses. CAB International, Wallingford

Boer DR et al (2014) Structural basis for DNA binding specificity by the auxin-dependent ARF transcription factors. Cell 156:577-589. https://doi.org/10.1016/j.cell.2013.12.027

Cass CL et al (2016) Cell wall composition and biomass recalcitrance differences within a genotypically diverse set of Brachypodium distachyon inbred lines. Front Plant Sci 7:708. https://doi.org/10. 3389/fpls.2016.00708

Chen J, Lausser A, Dresselhaus T (2014) Hormonal responses during early embryogenesis in maize. Biochem Soc Trans 42:325-331. https://doi.org/10.1042/BST20130260

Chen J, Strieder N, Krohn NG, Cyprys P, Sprunck S, Engelmann JC, Dresselhaus T (2017) Zygotic genome activation occurs shortly after fertilization in maize. Plant Cell 29:2106-2125. https://doi. org/10.1105/tpc. 17.00099

Egley GH, Paul RN Jr, Vaughn KC, Duke SO (1983) Role of peroxidase in the development of water-impermeable seed coats in Sida spinosa L. Planta 157:224-232. https://doi.org/10.1007/BF004 05186

Friml J et al (2003) Efflux-dependent auxin gradients establish the apical-basal axis of Arabidopsis. Nature 426:147-153. https:// doi.org/10.1038/nature02085

Gao Y et al (2021) The endosperm-specific transcription factor TaNAC019 regulates glutenin and strach accumulation and its elite allele improves wheat grain quality. Plant Cell. https://doi. org/10.1093/plcell/koaa040

Gijzen M, Van Huystee R, Buzzell RI (1993) Soybean seed coat peroxidase (a comparison of high-activity and low-activity genotypes). Plant Physiol 103:1061-1066. https://doi.org/10.1104/pp.103.4. 1061

Giorgino T (2009) Computing and visualizing dynamic time warping alignments in R: the dtw package. J Stat Softw 31:1-24. https:// doi.org/10.18637/jss.v031.i07

Griffiths JS, North HM (2017) Sticking to cellulose: exploiting Arabidopsis seed coat mucilage to understand cellulose biosynthesis and cell wall polysaccharide interactions. New Phytol 214:959_ 966. https://doi.org/10.1111/nph.14468

Guillon F et al (2012) A comprehensive overview of grain development in Brachypodium distachyon variety Bd21. J Exp Bot 63:739-755. https://doi.org/10.1093/jxb/err298

Haecker A, Gross-Hardt R, Geiges B, Sarkar A, Breuninger H, Herrmann M, Laux T (2004) Expression dynamics of WOX genes mark cell fate decisions during early embryonic patterning in Arabidopsis thaliana. Development 131:657-668. https://doi. org/10.1242/dev.00963

Hofmann F, Schon MA, Nodine MD (2019) The embryonic transcriptome of Arabidopsis thaliana. Plant Reprod 32:77-91. https://doi. org/10.1007/s00497-018-00357-2

Ito M, Sato Y, Matsuoka M (2002) Involvement of homeobox genes in early body plan of monocot. Int Rev Cytol 218:1-35. https://doi. org/10.1016/s0074-7696(02)18010-7

Itoh J et al (2005) Rice plant development: from zygote to spikelet. Plant Cell Physiol 46:23-47. https://doi.org/10.1093/pcp/pci501

Itoh J et al (2016) Genome-wide analysis of spatiotemporal gene expression patterns during early embryogenesis in rice. Development 143:1217-1227. https://doi.org/10.1242/dev.123661

Iwakawa $\mathrm{H}$ et al (2002) The ASYMMETRIC LEAVES2 gene of Arabidopsis thaliana, required for formation of a symmetric flat leaf lamina, encodes a member of a novel family of proteins characterized by cysteine repeats and a leucine zipper. Plant Cell Physiol 43:467-478. https://doi.org/10.1093/pcp/pcf077

Johri BM (1984) Embryology of angiosperms. Springer, Berlin, Heidelberg

Kamiya N, Nagasaki H, Morikami A, Sato Y, Matsuoka M (2003) Isolation and characterization of a rice WUSCHEL-type homeobox gene that is specifically expressed in the central cells of a quiescent center in the root apical meristem. Plant J 35:429-441. https://doi.org/10.1046/j.1365-313x.2003.01816.x

Kerstetter RA, Laudencia-Chingcuanco D, Smith LG, Hake S (1997) Loss-of-function mutations in the maize homeobox gene, knotted1, are defective in shoot meristem maintenance. Development 124:3045-3054

Kunieda T, Shimada T, Kondo M, Nishimura M, Nishitani K, HaraNishimura I (2013) Spatiotemporal secretion of PEROXIDASE36 is required for seed coat mucilage extrusion in Arabidopsis. Plant Cell 25:1355-1367. https://doi.org/10.1105/tpc.113.110072

Lee BH, Jeon JO, Lee MM, Kim JH (2015) Genetic interaction between GROWTH-REGULATING FACTOR and CUP-SHAPED COTYLEDON in organ separation. Plant Signal Behav. https://doi.org/ $10.4161 / 15592324.2014 .988071$

Leiboff S, Hake S (2019) Reconstructing the transcriptional ontogeny of maize and sorghum supports an inverse hourglass model of inflorescence development. Curr Biol 29(3410-3419):e3413. https://doi.org/10.1016/j.cub.2019.08.044

Levin M et al (2016) The mid-developmental transition and the evolution of animal body plans. Nature 531:637-641. https://doi.org/ 10.1038 /nature 16994

Liao CY, Smet W, Brunoud G, Yoshida S, Vernoux T, Weijers D (2015) Reporters for sensitive and quantitative measurement of auxin response. Nat Methods 12:207-210. https://doi.org/10.1038/ nmeth.3279

Long JA, Barton MK (1998) The development of apical embryonic pattern in Arabidopsis. Development 125:3027-3035

Mansfield SG, Briarty LG (1991) Early embryogenesis in Arabidopsis thaliana. II. The developing embryo. Can J Bot 69:461-476. https://doi.org/10.1139/b91-063

Mendu V et al (2011) Subfunctionalization of cellulose synthases in seed coat epidermal cells mediates secondary radial wall synthesis and mucilage attachment. Plant Physiol 157:441-453. https://doi. org/10.1104/pp.111.179069 
Moller B, Weijers D (2009) Auxin control of embryo patterning. Cold Spring Harb Perspect Biol 1:a001545. https://doi.org/10.1101/ cshperspect.a001545

Moller BK et al (2017) Auxin response cell-autonomously controls ground tissue initiation in the early Arabidopsis embryo. Proc Natl Acad Sci USA 114:E2533-E2539. https://doi.org/10.1073/ pnas. 1616493114

Nardmann J, Zimmermann R, Durantini D, Kranz E, Werr W (2007) WOX gene phylogeny in Poaceae: a comparative approach addressing leaf and embryo development. Mol Biol Evol 24:24742484. https://doi.org/10.1093/molbev/msm182

Nodine MD, Bartel DP (2012) Maternal and paternal genomes contribute equally to the transcriptome of early plant embryos. Nature 482:94-97. https://doi.org/10.1038/nature10756

O'Connor DL, Runions A, Sluis A, Bragg J, Vogel JP, Prusinkiewicz P, Hake S (2014) A division in PIN-mediated auxin patterning during organ initiation in grasses. PLoS Comput Biol 10:e1003447. https://doi.org/10.1371/journal.pcbi.1003447

Palovaara J, de Zeeuw T, Weijers D (2016) Tissue and organ initiation in the plant embryo: a first time for everything. Annu Rev Cell Dev Biol 32:47-75. https://doi.org/10.1146/annurev-cellb io-111315-124929

Patro R, Duggal G, Love MI, Irizarry RA, Kingsford C (2017) Salmon provides fast and bias-aware quantification of transcript expression. Nat Methods 14:417-419. https://doi.org/10.1038/nmeth. 4197

Qin Z et al (2017) Regulation of FT splicing by an endogenous cue in temperate grasses. Nat Commun 8:14320. https://doi.org/10. 1038/ncomms 14320

Quint M, Drost H-G, Gabel A, Ulrich KK, Bönn M, Grosse I (2012) A transcriptomic hourglass in plant embryogenesis. Nature 490:98101. https://doi.org/10.1038/nature11394

Radoeva T et al (2019) A robust auxin response network controls embryo and suspensor development through a basic helix loop helix transcriptional module. Plant Cell 31:52-67. https://doi.org/ $10.1105 /$ tpc. 18.00518

Robert HS et al (2013) Local auxin sources orient the apical-basal axis in Arabidopsis embryos. Curr Biol 23:2506-2512. https://doi.org/ 10.1016/j.cub.2013.09.039

Robert HS et al (2018) Maternal auxin supply contributes to early embryo patterning in Arabidopsis. Nat Plants 4:548-553. https:// doi.org/10.1038/s41477-018-0204-z

Robinson MD, McCarthy DJ, Smyth GK (2010) edgeR: a bioconductor package for differential expression analysis of digital gene expression data. Bioinformatics 26:139-140. https://doi.org/10. 1093/bioinformatics/btp616

Sarkar AK et al (2007) Conserved factors regulate signalling in Arabidopsis thaliana shoot and root stem cell organizers. Nature 446:811-814. https://doi.org/10.1038/nature05703

Schlereth A et al (2010) MONOPTEROS controls embryonic root initiation by regulating a mobile transcription factor. Nature 464:913-916. https://doi.org/10.1038/nature08836

Scholthof KBG, Irigoyen S, Catalan P, Mandadi KK (2018) Brachypodium: a monocot grass model genus for plant biology. Plant Cell 30:1673-1694. https://doi.org/10.1105/tpc.18.00083

Schon MA, Nodine MD (2017) Widespread contamination of Arabidopsis embryo and endosperm transcriptome data sets. Plant Cell 29:608-617. https://doi.org/10.1105/tpc.16.00845

Semiarti E, Ueno Y, Tsukaya H, Iwakawa H, Machida C, Machida Y (2001) The ASYMMETRIC LEAVES2 gene of Arabidopsis thaliana regulates formation of a symmetric lamina, establishment of venation and repression of meristem-related homeobox genes in leaves. Development 128:1771-1783

Shuai B, Reynaga-Pena CG, Springer PS (2002) The lateral organ boundaries gene defines a novel, plant-specific gene family. Plant Physiol 129:747-761. https://doi.org/10.1104/pp.010926
Smart MG, O'Brien TP (1983) The development of the wheat embryo in relation to the neighbouring tissues. Protoplasma 114:1-13. https://doi.org/10.1007/BF01279863

Smit ME, Weijers D (2015) The role of auxin signaling in early embryo pattern formation. Curr Opin Plant Biol 28:99-105. https://doi. org/10.1016/j.pbi.2015.10.001

Smith LG, Jackson D, Hake S (1995) Expression of knotted1 marks shoot meristem formation during maize embryogenesis. Dev Genet 16:344-348. https://doi.org/10.1002/dvg.1020160407

Takaiwa F, Yamanouchi U, Yoshihara T, Washida H, Tanabe F, Kato A, Yamada K (1996) Characterization of common cis-regulatory elements responsible for the endosperm-specific expression of members of the rice glutelin multigene family. Plant Mol Biol 30:1207-1221. https://doi.org/10.1007/BF00019553

ten Hove CA, Lu KJ, Weijers D (2015) Building a plant: cell fate specification in the early Arabidopsis embryo. Development 142:420 430. https://doi.org/10.1242/dev.111500

The International Brachypodium Initiative. Pi Vogel J et al (2010) Genome sequencing and analysis of the model grass Brachypodium distachyon. Nature 463:763-768. https://doi.org/10.1038/ nature 08747

Tsuda K, Hake S (2016) Homeobox transcription factors and the regulation of meristem development and maintenance. In: Gonzalez DH (ed) Plant transcription factors. Academic Press, Cambridge, pp 215-228

Ulmasov T, Murfett J, Hagen G, Guilfoyle TJ (1997) Aux/IAA proteins repress expression of reporter genes containing natural and highly active synthetic auxin response elements. Plant Cell 9:1963-1971. https://doi.org/10.1105/tpc.9.11.1963

Ursache R, Andersen TG, Marhavy P, Geldner N (2018) A protocol for combining fluorescent proteins with histological stains for diverse cell wall components. Plant J 93:399-412. https://doi.org/10.1111/ tpj. 13784

Vaddepalli P, Zeeuw T, Strauss S, Bürstenbinder K, Liao C-Y, Smith R, Weijers D (2021) A molecular framework for control of oriented cell division in the Arabidopsis embryo. bioRxiv. https://doi.org/ 10.1101/2021.02.09.430440

Venglat P et al (2011) Gene expression analysis of flax seed development. BMC Plant Biol 11:74. https://doi.org/10.1186/ 1471-2229-11-74

Xiang D et al (2011b) POPCORN functions in the auxin pathway to regulate embryonic body plan and meristem organization in Arabidopsis. Plant Cell 23:4348-4367. https://doi.org/10.1105/ tpc.111.091777

Xiang D et al (2011a) Genome-wide analysis reveals gene expression and metabolic network dynamics during embryo development in Arabidopsis. Plant Physiol 156:346-356. https://doi.org/10.1104/ pp.110.171702

Xiang D et al (2019) The transcriptional landscape of polyploid wheats and their diploid ancestors during embryogenesis and grain development. Plant Cell 31:2888-2911. https://doi.org/10.1105/tpc.19. 00397

Yang S, Johnston N, Talideh S, Mitchell S, Jeffree C, Goodrich J, Ingram G (2008) The endoserm-specific ZHOUPI gene of Arabidopsis thaliana regulates endosperm breakdown and embryonic epidermal development. Development 135:3501-3509. https://doi. org/10.1242/dev.026708

Yi F et al (2019) High temporal-resolution transcriptome landscape of early maize seed development. Plant Cell 31:974-992. https://doi. org/10.1105/tpc.18.00961

Yoshida S, Barbier de Reuille P, Lane B, Bassel GW, Prusinkiewicz P, Smith RS, Weijers D (2014) Genetic control of plant development by overriding a geometric division rule. Dev Cell 29:75-87. https://doi.org/10.1016/j.devcel.2014.02.002 
Yu G, Wang LG, Han Y, He QY (2012) clusterProfiler: an R package for comparing biological themes among gene clusters. OMICS 16:284-287. https://doi.org/10.1089/omi.2011.0118

Zhao P, Begcy K, Dresselhaus T, Sun MX (2017) Does early embryogenesis in eudicots and monocots involve the same mechanism and molecular players? Plant Physiol 173:130-142. https://doi. org/10.1104/pp.16.01406
Publisher's Note Springer Nature remains neutral with regard to jurisdictional claims in published maps and institutional affiliations. 